\title{
The Exterior Neumann Problem for the Three-Dimensional Helmholtz Equation
}

\author{
ERgun Ar \& R. E. KLEINMAN \\ Communicated by J. MeIXNER
}

\section{Introduction}

A method for explicitly solving the exterior Dirichlet problem for the threedimensional Helmholtz equation in terms of the Dirichlet Green's function for Laplace's equation has recently been found [6]. The present work shows how a similar technique may be used to solve the exterior Neumann problem in terms of the corresponding Neumann-Green function for Laplace's equation.

The existence of the required potential Green's function is proven by KeLLOGG [5]. This work formed a basis for WEYL [14] and MüLLER [9] who proved that a solution of the exterior Dirichlet problem for the Helmholtz equation exists. LEIS [7] extended these ideas to establish the existence of a solution of the exterior Neumann problem for the Helmholtz equation and an alternative existence proof has recently been given by WERNER [13]. The utility of these results in actually producing a solution, however, has not been demonstrated.

That the solution of the boundary value problem for the Helmholtz equation may be found as a perturbation of the solution of the corresponding potential problem is shown by NOBLE [10]. The solution is expressed as a power series in wave number, each term of which is the solution of an integral equation of the second kind which differs from term to term only in its inhomogeneous part. This formulation of the problem does not yield an explicit representation of the solution, however, except as the formal inverse of an integral operator.

In the present work, a representation of the desired solution is derived which expresses the solution as a linear operation on itself plus a known term. The linear operator is shown to be bounded, and the inverse, for small values of wave number, is given by a standard Liouville-Neumann series.

The principal result of the paper is contained in the following.

Theorem. If

(a) $V$ is the volume exterior to $B$, a smooth, closed, bounded surface in $E^{3}$.

(b) $G_{0}\left(p, p_{1}\right)$ is the potential Green's function of the second kind for this surface

$$
\left(\frac{\partial}{\partial n} G_{0}\left(p_{B}, p_{1}\right)=0\right),
$$


and

(c) $u$ is a solution of the Helmholtz equation $\left(\nabla^{2}+k^{2}\right) u=0, p \in \bar{V}$, and $u$ also satisfies the radiation condition at infinity, then, for $|k|$ sufficiently small, $u$ is given explicitly in terms of the values of its normal derivative on $B$ as

$$
u=e^{i k r} \sum_{n=0}^{\infty} L^{n} \circ u^{(0)}
$$

where the operator $L$ is defined by

$$
\begin{aligned}
\omega \rightarrow L \circ w= & -2 i k \int_{V} \frac{G_{0}\left(p, p_{1}\right)}{r_{1}} \frac{\partial}{\partial r_{1}}\left[r_{1} \omega\left(p_{1}\right)\right] d v_{1}+ \\
& +i k \int_{B} G_{0}\left(p, p_{B}\right) \frac{\partial r_{B}}{\partial n} \omega\left(p_{B}\right) d \sigma_{B},
\end{aligned}
$$

and

$$
u^{(0)}=-\frac{1}{4 \pi} \int_{B} G_{0}\left(p, p_{B}\right) e^{-i k r_{B}} \frac{\partial u}{\partial n} d \sigma_{B}
$$

$p$ and $p_{1}$ denote points in $V, p_{B}$ a point on $B$ at distances $r, r_{1}$, and $r_{B}$ respectively from the origin of a coordinate system centered in $B, d v_{1}$ is a volume element in coordinates $p_{1}, d \sigma_{B}$ a surface element in coordinates $p_{B}$, and $\partial / \partial n$ is the normal derivative directed out of $V$.

If the value of $\partial u / \partial n$ is specified and the static Green's function $G_{0}$ is known, then clearly $u^{(0)}$ is also known explicitly.

In the following section we introduce notation and derive an integral representation of functions regular at infinity. This theorem is then used, in Section 3, to obtain a representation of functions satisfying the Helmholtz equation and a Sommerfeld radiation condition. It is shown that the result includes the cases when $u$ represents the velocity potential of an acoustic field scattered by a rigid surface when illuminated by plane waves or point sources. In Section 4 , we prove that the representation considered as an operator equation may be solved iteratively, thus establishing the main theorem. The relation between the series of iterates and the usual low frequency expansion is described. The method is demonstrated in Section 5 in the example of scattering of a plane wave of sound by a rigid sphere. The results are shown to agree with the classical solution.

Some deficiencies in the treatment of the Dirichlet problem are pointed out and corrected. No fundamental error has been found, however, and the treatment here parallels that in [6] as closely as possible, thus eliminating the repetition of many detailed arguments.

\section{A Representation Theorem}

The notation we use is consistent with that in [6]. Thus $B$ is the boundary of a smooth closed bounded surface in $E^{3}$, and $V$ is the volume exterior to $B$. Erect a spherical polar coordinate system with origin interior to $B$, and denote by $p$ a point $(r, \vartheta, \varphi)$ in $V$ and by $p_{B}$ a point $\left(r_{B}, \vartheta_{B}, \varphi_{B}\right)$ on $B$. The distance between 
any two points $p, p_{1} \in \bar{V}=V \cup B$ will be denoted by $R\left(p, p_{1}\right)$ and is defined as

$$
R\left(p, p_{1}\right)=\left[r^{2}+r_{1}^{2}-2 r r_{1}\left(\cos \vartheta \cos \vartheta_{1}+\sin \vartheta \sin \vartheta_{1} \cos \left(\varphi-\varphi_{1}\right)\right)\right]^{\frac{1}{2}} .
$$

Furthermore, let $c=\max r_{B}$, so that $B$ is contained in a sphere of radius $c$ and assume that the normal to $B$ is directed inward (out of $V$ ).

A more precise definition of the degree of smoothness of the surface $B$ is provided by requiring that it be described by an equation

$$
r_{B}=g(\vartheta, \varphi)
$$

where $g$ is a continuously differentiable function of $\vartheta$ and $\varphi$. Moreover, the quantity $\hat{n} \cdot \hat{r}_{B}$, where $\hat{n}$ is the unit normal and $\hat{r}_{B}$ the unit radius vector, must be uniformly Hölder continuous on the surface. All of the results to follow remain valid if $B$ is a finite union of disjoint surfaces, provided each of them satisfies the above requirements.

A real valued function $f: V \rightarrow E^{1}$ is defined to be regular (in the sense of KELLOGG) at infinity if

$$
r f=O(1), \quad r^{2} \frac{\partial f}{\partial r}=O(1) \quad \text { as } r \rightarrow \infty
$$

uniformly in $\vartheta$ and $\varphi$.

A complex valued function is regular if both real and imaginary parts are regular.

The Neumann potential Green's function for the surface $B$, the existence and uniqueness of which is proven by KellogG, is defined to be a function $G_{0}\left(p, p_{1}\right)$ of two points and may be written in the form

$$
G_{0}\left(p, p_{1}\right)=-\frac{1}{4 \pi R\left(p, p_{1}\right)}+u_{0}\left(p, p_{1}\right), \quad p, p_{1} \in \bar{V}
$$

where $u_{0}\left(p, p_{1}\right)$ has no singularities in $\bar{V}$ and

(a) $\nabla^{2} u_{0}\left(p, p_{1}\right)=0, \quad p, p_{1} \in V$,

(b) $\frac{\partial}{\partial n} G_{0}\left(p_{B}, p_{1}\right)=0$.

[This notation is used repeatedly and has the following meaning. Let $\nabla$ be the gradient operating on coordinates of $p$ and $\hat{n}$ the unit normal on $B$ directed out of $V$. Then define $\nabla G_{0}\left(p_{B}, p_{1}\right)=\left.\nabla G_{0}\left(p, p_{1}\right)\right|_{p \in B}$ and $\frac{\partial}{\partial n} G_{0}\left(p_{B}, p_{1}\right)=\hat{n}$. $\nabla G_{0}\left(p_{B}, p_{1}\right)$.]

(c) $u_{0}$ is regular at infinity.

In terms of this Green's function we may state an integral representation of functions regular at infinity. This is contained in

Theorem 2.1. If $\omega: \bar{V} \rightarrow E^{1}$ is a function which is twice differentiable in $V$ and regular at infinity, then

$$
\omega(p)=\int_{V} G_{0}\left(p, p_{1}\right) \nabla^{2} \omega\left(p_{1}\right) d v_{1}-\int_{B} G_{0}\left(p, p_{B}\right) \frac{\partial}{\partial n} \omega\left(p_{B}\right) d \sigma_{B}
$$


where $G_{0}\left(p, p_{1}\right)$ is the Neumann potential Green's function, $d v_{1}$ is the volume element and $\nabla^{2}$ is the Laplacian both expressed in coordinates $\left(r_{1}, \vartheta_{1}, \varphi_{1}\right), d \sigma_{B}$ is the surface element and $\partial / \partial n$ the inward normal derivative (out of $V$ ) both expressed in coordinates $\left(r_{B}, \vartheta_{B}, \varphi_{B}\right)$.

Proof. Let $V^{\prime}$ denote the volume exterior both to $B$ and a small sphere $S$ centered at $R\left(p, p_{1}\right)=0$, and interior to a large sphere, $B_{1}$, entirely enclosing $B$. Then by Green's second identity

$$
\begin{aligned}
\int_{V^{\prime}}\left[\omega\left(p_{1}\right) \nabla^{2}\right. & \left.G_{0}\left(p, p_{1}\right)-G_{0}\left(p, p_{1}\right) \nabla^{2} \omega\left(p_{1}\right)\right] d v_{1} \\
\quad & \int_{B+B_{1}+S}\left[\omega\left(p^{\prime}\right) \frac{\partial}{\partial n} G_{0}\left(p, p^{\prime}\right)-G_{0}\left(p, p^{\prime}\right) \frac{\partial}{\partial n} \omega\left(p^{\prime}\right)\right] d \sigma^{\prime} .
\end{aligned}
$$

Letting the radius of $S$ shrink to 0 , we find that

$$
\omega(p)=-\int_{S}\left(\omega \frac{\partial G_{0}}{\partial n}-G_{0} \frac{\partial \omega}{\partial n}\right) d \sigma^{\prime} .
$$

If we make use of this result together with the differential equation and boundary condition satisfied by $G_{0}$, equation (2.7) becomes

$$
\begin{aligned}
\omega(p)= & \int_{V^{\prime}} G_{0}\left(p, p_{1}\right) \nabla^{2} \omega\left(p_{1}\right) d v_{1}-\int_{B} G_{0}\left(p, p_{B}\right) \frac{\partial}{\partial n} \omega\left(p_{B}\right) d \sigma_{B}+ \\
& +\int_{B_{1}}\left[\omega\left(p_{B_{1}}\right) \frac{\partial}{\partial n} G_{0}\left(p, p_{B_{1}}\right)-G_{0}\left(p, p_{B_{1}}\right) \frac{\partial \omega\left(p_{B_{1}}\right)}{\partial n}\right] d \sigma_{B_{1}} .
\end{aligned}
$$

The integral over $B_{1}$ vanishes as the radius of $B_{1}$ becomes infinite by virtue of the regularity of $\omega$ and $G_{0}$ (details are given in [6]), thus proving the theorem.

\section{A Representation of Wave Functions}

A function $u: \bar{V} \rightarrow E^{1}$ is a scalar wave function for the volume $V$ if

(a) $u(p)$ is twice continuously differentiable in $\bar{V}\left(u \in C^{2}(\bar{V})\right)$ with the understanding that if $p \in B$ the limit is taken from the exterior, $V$,

(b) $\left(\nabla^{2}+k^{2}\right) u(p)=0, \quad p \in \bar{V}$,

(c) $r\left(\frac{\partial u}{\partial r}-i k u\right)=o(1), \quad$ as $r \rightarrow \infty$, uniformly in $\vartheta$ and $\varphi$.

Other statements of the radiation condition are possible (WILCOX, [16]), but this form, as given originally by SoMmERFELD [11] is quite adequate for our purposes; it may be stronger than necessary, but it does what we want it to do, namely, characterize radiating solutions of the Helmholtz equation.

We wish to employ Theorem 2.1 to represent scalar wave functions, but they are not regular at infinity in the sense of KeLLOGG. In order to modify them, we employ a well-known expansion theorem given with varying restriction by ATKINSON [1], SOMMERFELd [12], BARRAR \& KAY [2] and most generally by WILCOX [15]: 
Theorem 3.1. If $u$ is a scalar wave function for the volume $V$, then

$$
u=\frac{e^{i k r}}{r} \sum_{n=0}^{\infty} \frac{f_{n}(\vartheta, \varphi)}{r^{n}},
$$

where the series converges absolutely and uniformly for $r \geqq c+\varepsilon, \varepsilon>0$ and $c=\max r_{B}$. Furthermore, the series may be differentiated term by term with respect to $r, \vartheta$, and $\varphi$ any number of times, and the resulting series all converge absolutely and uniformly.

It follows from this expansion theorem that if $u$ is a scalar wave function, then

$$
\tilde{u}(p)=e^{-i k r} u(p)
$$

is regular and satisfies the hypothesis of Theorem 2.1. Furthermore, as may be verified easily, since $u$ satisfies the Helmholtz equation,

$$
\nabla^{2} \tilde{u}=-\frac{2 i k}{r} \frac{\partial}{\partial r}(r \tilde{u})
$$

In addition,

$$
\frac{\partial}{\partial n} \tilde{u}\left(p_{B}\right)=\hat{n} \cdot \nabla \tilde{u}=\hat{n} \cdot \nabla e^{-i k r} u=-i k \hat{n} \cdot \hat{r}_{B} \tilde{u}\left(p_{B}\right)+e^{-i k r_{B}} \frac{\partial u\left(p_{B}\right)}{\partial n},
$$

where $\hat{n}$ and $\hat{r}_{B}$ are unit vectors in the normal (inward) and radial directions respectively at $p_{B}$. (If $B$ is a sphere, $\hat{n}=-\hat{r}_{B}$.) Incorporating these results in the representation theorem, Theorem 2.1, establishes the following:

Theorem 3.2. If

(a) $u$ is a scalar wave function for $V$, the exterior of a smooth, closed, bounded surface $B$ and

(b) $G_{0}\left(p, p_{1}\right)$ is the Neumann potential Green's function for this surface

$$
\left(\frac{\partial}{\partial n} G_{0}\left(p_{B}, p_{1}\right)=0\right),
$$

then $\tilde{u}\left(=e^{-i k r} u\right)$ may be represented as

$$
\begin{aligned}
\tilde{u}(p)= & -2 i k \int_{V} \frac{G_{0}\left(p, p_{1}\right)}{r_{1}} \frac{\partial}{\partial r_{1}}\left[r_{1} \tilde{u}\left(p_{1}\right)\right] d v_{1}+ \\
& +i k \int_{B} G_{0}\left(p, p_{B}\right) \hat{n} \cdot \hat{r}_{B} \tilde{u}\left(p_{B}\right) d \sigma_{B}-\int_{B} G_{0}\left(p, p_{B}\right) e^{-i k r_{B}} \frac{\partial u\left(p_{B}\right)}{\partial n} d \sigma_{B} .
\end{aligned}
$$

Included in this theorem are representations of the solutions of the two most common exterior Neumann problems for the Helmholtz equation and the surface $B$. If

$$
\frac{\partial u}{\partial n}=\frac{\partial}{\partial n}\left[\frac{e^{i k R\left(p_{B}, p_{0}\right)}}{4 \pi R\left(p_{B}, p_{0}\right)}\right],
$$


then $u$ represents the regular part of the Neumann Green's function for the Helmholtz equation. If

$$
\frac{\partial u}{\partial n}=-\frac{\partial}{\partial n}\left(e^{i k \vec{r} \cdot \hat{a}}\right)
$$

then $u$ represents the field scattered when a plane acoustic wave is incident in the direction $\hat{\alpha}$ on a rigid surface $B$. Note that the representation, (3.6), is in terms of $u$ but $\tilde{u}$ is easily found by multiplying with the phase factor $e^{i k \mathbf{r}}$.

The difference between the representation (3.6) and the corresponding expression in the Dirichlet case is the occurrence of a surface integral involving the unknown function as well as one involving its normal derivative on the boundary. We shall show that the term involving the unknown function can be lumped with the volume integral leading to an expression of the form

$$
\tilde{u}=L \circ \tilde{u}+u^{(0)}
$$

which may be inverted exactly as in [6]. This is done in the following section.

\section{A Liouville-Neumann Expansion}

The form of (3.9) suggests that an explicit solution may be given in the form of a Liouville-Neumann series, i.e.,

$$
\tilde{u}=(I-L)^{-1} \circ u^{(0)}=\sum_{n=0}^{\infty} L^{n} \circ u^{(0)} .
$$

That this, in fact, is the case and that the series does converge to the solution is the subject of this section. The proof parallels the treatment of the Dirichlet case, though some modifications are necessary.

First we define the partial sums

$$
u^{(N)}=\sum_{n=0}^{N} L^{n} \circ u^{(0)}
$$

We shall show that $\tilde{u}$ (the solution we seek), $u^{(0)}$ (the known term), and all the iterates $u^{(N)}, N \geqq 1$, are elements of a normed linear vector space and that in this norm

$$
\lim _{N \rightarrow \infty} u^{(N)}=\tilde{u} .
$$

Recall that $V$ is the volume exterior to the surface $B$, and let $\delta$ be the radius of a sphere entirely containing $B$ in its interior. With the surface $B$ we associate the following function space $W$ consisting of the set of functions $\omega: V \rightarrow E^{1}$ satisfying

$$
\begin{gathered}
\omega \in C^{2}(V), \quad \omega \in C^{1}(\bar{V}), \\
\omega=\frac{1}{r} \sum_{n=0}^{\infty} \frac{f_{n}(\vartheta, \varphi)}{r^{n}}, \quad r \geqq \delta,
\end{gathered}
$$


and the series is uniformly and absolutely convergent and term by term differentiable with respect to $r, \vartheta$ and $\varphi$ as are the derived series,

$$
f_{n}=\sum_{m=n}^{\infty} Y_{m}(\vartheta, \varphi)
$$

where $Y_{m}$ is an $m^{\text {th }}$ order spherical harmonic, i.e.,

$$
Y_{m}(\vartheta, \varphi)=\sum_{l=-m}^{m} A_{l m} P_{m}^{l}(\cos \vartheta) e^{i l \varphi}
$$

Clearly $W$ is a linear space. It is essentially the same function space introduced in the treatment of the Dirichlet problem, although the present characterization is more precise.

We define the following norm on $W$,

$$
\|\omega\|=\max _{p \in \bar{V}}|\omega(p)|
$$

We now proceed to prove a series of lemmas which enable us to establish the main result. We define two linear operators, $K$ and $K_{1}$, as

and

$$
\omega \rightarrow K \circ \omega=-2 i k \int_{V} \frac{G_{0}\left(p, p_{1}\right)}{r_{1}} \frac{\partial}{\partial r_{1}}\left[r_{1} \omega\left(p_{1}\right)\right] d v_{1},
$$

$$
\omega \rightarrow K_{1} \circ \omega=i k \int_{B} G_{0}\left(p, p_{B}\right) \hat{n} \cdot \hat{r}_{B} \omega\left(p_{B}\right) d \sigma_{B} .
$$

The equation we wish to solve then is

where

$$
\tilde{u}=L \circ \tilde{u}+u^{(0)}
$$

$$
u^{(0)}=-\int_{B} G_{0}\left(p, p_{B}\right) e^{-i r_{B}} \frac{\partial u\left(p_{B}\right)}{\partial n} d \sigma_{B}
$$

and

$$
L \circ \tilde{u}=K \circ \tilde{u}+K_{1} \circ \tilde{u} .
$$

Since $L$ is linear and $K$ is the same operator dealt with in [6], the decomposition enables us to cite the already known properties of $K$. The static Green's function involved is, of course, different, but this does not change the behavior at infinity or at the source which are the vital properties. The different boundary condition does require a slightly more detailed proof of Lemma 4.4 below. We still must establish the properties of $K_{1}$, but happily this is much simpler. The first task, however, is to show that $u^{(0)}$ is in $W$.

Lemma 4.1. $u^{(0)} \in W$.

Proof. The definition of $u^{(0)},(4.9)$, shows that $u^{(0)}$ consists of the potential of a single layer distribution of density $e^{-i k r_{B}} \frac{\partial u}{\partial n}$ plus another term (corre- 
sponding to the regular part of the Green's function) which is at least as well behaved. Therefore the differentiability of $u^{(0)}$ is essentially that of the potential. If the density $e^{-i k r_{B}} \frac{\partial u}{\partial n}$ is piecewise continuous, then according to KELLOGG $[5$, p. 122] the potential is infinitely differentiable in $V$, thus, in particular, the potential (hence $u^{(0)}$ ) is twice continuously differentiable in $V$. Furthermore, if the density is uniformly Hölder continuous, then again, according to KELLOGG $\left[5\right.$, p. 165] the potential (hence $u^{(0)}$ ) is continuously differentiable in the closure $\bar{V}$. That the density is uniformly Hölder continuous (hence piecewise continuous as well) is implied by the fact that $u$ is a scalar wave function, and therefore $u \in C^{2}(\bar{V})$. This, in turn, implies $\frac{\partial u}{\partial n} \in C^{1}(B)$, which together with the fact that $B$ is closed ensures that $\partial u / \partial n$ is uniformly Hölder continuous on $B$. Since $r_{B}$ is a continuously differentiable function of $\vartheta$ and $\varphi$ it follows that $e^{-i k r_{B}}$ is uniformly Hölder continuous, hence so is the product $e^{-i k r_{B}} \frac{\partial u}{\partial n}$. Therefore $u^{(0)}$ satisfies (4.4a).

$G_{0}\left(p, p_{B}\right)$ may be expanded in an absolutely and uniformly convergent series of spherical harmonics of the form (e.g. KELLOGG [5, p. 143]),

$$
G_{0}\left(p, p_{B}\right)=\sum_{n=0}^{\infty} \sum_{m=-n}^{n} A_{m n}\left(p_{B}\right) \frac{1}{r^{n+1}} P_{n}^{m}(\cos \vartheta) e^{i m \varphi} \quad r \geqq \delta .
$$

Thus for $r \geqq \delta$ we may rewrite $u^{(0)}$ as

$$
u^{(0)}=-\sum_{n=0}^{\infty} \frac{1}{r^{n+1}} \sum_{m=-n}^{n} P_{n}^{m}(\cos \vartheta) e^{\mathrm{im} \varphi} \int_{B} A_{m n}\left(p_{B}\right) e^{-i k r_{B}} \frac{\partial u}{\partial n} d \sigma_{B}
$$

which is of the form

$$
u^{(0)}=\sum_{n=0}^{\infty} \frac{Y_{n}(\vartheta, \varphi)}{r^{n+1}}
$$

Thus (4.4b) and (4.4c) are also satisfied and the lemma is proven.

Lemma 4.2. If $\omega \in W$, then $L \circ \omega \in W$.

Proof. Since $L=K+K_{1}$ (all linear) and $W$ is a linear space, it suffices to show that $\omega \in W$ implies that $K \circ \omega \in W$ and $K_{1} \circ \omega \in W$. That $\omega \in W$ implies $K \circ \omega \in W$ is proven in [6]. (The fact that the potential of a volume distribution is differentiable up to and including the boundary is proven by KeLLOGG [5, p. 152]. This is needed for the second part of (4.4a) which was omitted in [6].) It remains to show that $\omega \in W$ implies $K_{1} \circ \omega \in W$. Equation (4.7) shows that $K_{1} \circ \omega$ is the potential of a single layer distribution of density $i k \hat{n} \cdot \hat{r}_{B} \omega\left(p_{B}\right)$ plus a regular term. That is

$$
K_{1} \circ \omega=i k \int_{B}\left[-\frac{1}{4 \pi R\left(p, p_{B}\right)}+u_{0}\left(p, p_{B}\right)\right] \hat{n} \cdot \hat{r}_{B} \omega\left(p_{B}\right) d \sigma_{B},
$$

where $u_{0}$ is a regular potential function at all points $p \in \bar{V}$. The proof is exactly the same as that used to prove Lemma 4.1, provided the density $i k \hat{n} \cdot \hat{r}_{B} \omega\left(p_{B}\right)$ 
is uniformly Hölder continuous. The fact that $\omega \in W$ ensures that $\omega \in C^{1}(V)$. With the fact that $B$ is closed and finite, it follows that $\omega\left(p_{B}\right)$ is uniformly Hölder continuous. The density then will be uniformly Hölder continuous if $\hat{n} \cdot \hat{r}_{B}$ is. This, however, is one of the smoothness requirements on $B$ (Section 2). Provided then that the surface is sufficiently smooth in this sense, the fact that $K_{1} \circ \omega \in W$ follows by the same arguments used to establish Lemma 4.1.

Observe that this proof makes no use of the factor $k$ in the definition of the operators. Thus if we define new operators, $O, O_{1}$, and $L_{1}$, all independent of $k$, so that

where

$$
L=k L_{1}=k O+k O_{1}
$$

and

$$
O \circ \omega=-2 i \int_{V} \frac{G_{0}\left(p, p_{1}\right)}{r_{1}} \frac{\partial}{\partial r_{1}}\left[r_{1} \omega\left(p_{1}\right)\right] d v_{1}
$$

$$
O_{1} \circ \omega=i \int_{B} G_{0}\left(p, p_{B}\right) \hat{n} \cdot \hat{r}_{B} \omega\left(p_{B}\right) d \sigma_{B},
$$

then it follows immediately that

Lemma 4.3. $\omega \in W$ implies that $L_{1} \circ \omega \in W$.

Lemma 4.4. $L_{1}$ is bounded.

Proof. It is sufficient to show that there exists $M<\infty$ such that if $\omega \in W$, then $\left\|L_{1} \circ \omega\right\| \leqq M\|\omega\|$. Furthermore, since

and

$$
L_{1}=O+O_{1}
$$

$$
\left\|L_{1} \circ \omega\right\|=\left\|O \circ \omega+O_{1} \circ \omega\right\| \leqq\|O \circ \omega\|+\left\|O_{1} \circ \omega\right\|,
$$

it is sufficient to demonstrate that

and

$$
\|O \circ \omega\| \leqq M_{1}\|\omega\|, \quad M_{1}<\infty,
$$

$$
\left\|O_{1} \circ \omega\right\| \leqq M_{2}\|\omega\|, \quad M_{2}<\infty .
$$

Consider (4.20) first. Here the proof is slightly more complicated than that given in [6] since the static Green's function does not vanish on $B$, but the procedure is much the same. Note that $\frac{\partial f_{0}}{\partial r_{1}} \equiv 0$, where $f_{0}$ is the first coefficient in the expansion of $\omega$, equation (4.4b). Thus we may write

or, equivalently,

$$
O \circ \omega=-2 i \int_{V} \frac{G_{0}\left(p, p_{1}\right)}{r_{1}} \frac{\partial}{\partial r_{1}}\left[r_{1} \omega\left(p_{1}\right)-f_{0}\right] d v_{1}
$$

$$
\begin{aligned}
O \circ \omega= & -2 i \int_{V} \frac{1}{r_{1}^{2}} \frac{\partial}{\partial r_{1}}\left(r_{1} G_{0}\left(p, p_{1}\right)\left[r_{1} \omega\left(p_{1}\right)-f_{0}\right]\right) d v_{1}+ \\
& +2 i \int_{V} \frac{1}{r_{1}}\left[\omega\left(p_{1}\right)-\frac{f_{0}}{r_{1}}\right] \frac{\partial}{\partial r_{1}}\left[r_{1} G_{0}\left(p, p_{1}\right)\right] d v_{1}
\end{aligned}
$$


It is a trivial calculation to show that for any scalar function $F$

$$
\frac{1}{r_{1}^{2}} \frac{\partial}{\partial r_{1}}\left(r_{1}^{2} F\right)=\nabla_{1} \cdot\left(\hat{r}_{1} F\right)
$$

where $\nabla_{1}$ operates on $\left(r_{1}, \vartheta_{1}, \varphi_{1}\right)$. With this relation, the divergence theorem may be employed to transform the first integral on the right-hand side of (4.22) yielding

$$
\begin{aligned}
O \circ \omega= & -2 i \int_{B} \hat{n} \cdot \hat{r}_{B} G_{0}\left(p, p_{B}\right)\left[\omega\left(p_{B}\right)-\frac{f_{0}}{r_{B}}\right] d \sigma_{B}+ \\
& +2 i \int_{V} \frac{1}{r_{1}}\left[\omega\left(p_{1}\right)-\frac{f_{0}}{r_{1}}\right] \frac{\partial}{\partial r_{1}}\left[r_{1} G_{0}\left(p, p_{1}\right)\right] d v_{1} .
\end{aligned}
$$

An additional surface integral over a sphere of infinite radius vanishes because

$$
r_{1}^{2} G_{0}\left(\omega-\frac{f_{0}}{r_{1}}\right)=o(1) \quad \text { as } r_{1} \rightarrow \infty \text {. }
$$

The volume integral in (4.23) is the same as that arising in [6] except for the different definition of $G_{0}$ and a factor $1 / r_{1}$. Actually this factor should have been present there also, though its absence does not affect the conclusions.

Now we employ the following estimate

$$
\left|\omega(p)-\frac{f_{0}}{r}\right| \leqq \frac{C}{r^{2}}\|\omega\|, \quad p \in \bar{V}
$$

where $C$ is some constant independent of $\omega$. A different form of this was derived in [6], and, although the proof given there is subject to question, the result as stated above is still valid. A rigorous demonstration of this will be included in a subsequent communication. Assuming the validity of this estimate, together with the fact that since $\hat{n}$ and $\hat{r}_{B}$ are unit vectors, $\left|\hat{n} \cdot \hat{r}_{B}\right| \leqq 1$, we see that for $p \in \bar{V}$

$$
|O \circ \omega| \leqq\left[\int_{B}\left|G_{0}\left(p, p_{B}\right)\right| \frac{1}{r_{B}^{2}} d \sigma_{B}+\int_{V} \frac{1}{r_{1}^{3}}\left|\frac{\partial}{\partial r_{1}}\left(r_{1} G_{0}\right)\right| d v_{1}\right] 2 C\|\omega\| .
$$

The surface integral may be rewritten, separating out the singular part of the Green's function, as

$$
\int_{B}\left|G_{0}\left(p, p_{B}\right)\right| \frac{1}{r_{B}^{2}} d \sigma_{B} \leqq \int_{B} \frac{1}{4 \pi r_{B}^{2} R\left(p, p_{B}\right)} d \sigma_{B}+\int_{B}\left|u_{0}\left(p, p_{B}\right)\right| \frac{1}{r_{B}^{2}} d \sigma_{B} .
$$

The first term on the right is the potential of a single layer distribution of density $1 / 4 \pi r_{B}^{2}$. Since $r_{B} \neq 0$ (the origin was taken within $B$ ) and the surface is smooth, closed and finite, this density is uniformly Hölder continuous which means (KellogG $[5$, p. 165]) that the potential is continuously differentiable for all points $p \in \bar{V}$. The second term on the right hand side of (4.25) is the integral of a bounded function over a finite surface and hence is also bounded. Thus for 
some $N<\infty$,

$$
\int_{B}\left|G_{0}\left(p, p_{B}\right)\right| \frac{1}{r_{B}^{2}} d \sigma_{B}<N, \quad p \in \bar{V} .
$$

The volume integral in (4.24) is also bounded since the integrand is sufficiently well behaved. At the singularity of $G_{0}$

$$
\frac{1}{r_{1}^{3}}\left|\frac{\partial}{\partial r_{1}}\left(r_{1} G_{0}\right)\right|=O\left(1 / R^{2}\right) \quad \text { as } R \rightarrow 0
$$

and is therefore integrable over any finite volume containing the singularity (KellogG [5, p. 148]). Furthermore

$$
\frac{1}{r_{1}^{3}}\left|\frac{\partial}{\partial r_{1}}\left(r_{1} G_{0}\right)\right|=O\left(1 / r_{1}^{5}\right) \quad \text { as } r_{1} \rightarrow \infty,
$$

thus for some $N_{1}<\infty$

$$
\int_{V} \frac{1}{r_{1}^{3}}\left|\frac{\partial}{\partial r_{1}}\left[r_{1} G_{0}\left(p, p_{1}\right)\right]\right| d v_{1}<N_{1}, \quad p \in \bar{V} .
$$

With (4.24), (4.26) and (4.27) we have

$$
|O \circ \omega| \leqq\left(N+N_{1}\right) 2 C\|\omega\|, \quad p \in \bar{V} .
$$

In particular this is true for the maximum value of $|O \circ \omega|$, therefore, renaming the constants,

$$
\|O \circ \omega\| \leqq M_{1}\|\omega\| \text {. }
$$

Next we establish (4.21), thus proving the lemma. With the definition of $O_{1}$ (Equation 4.17) we see that

By definition,

$$
\left|O_{1} \circ \omega\right| \leqq \int_{B}\left|G_{0}\left(p, p_{B}\right)\right|\left|\hat{n} \cdot \hat{r}_{B}\right|\left|\omega\left(p_{B}\right)\right| d \sigma_{B} .
$$

$$
\left|\omega\left(p_{B}\right)\right| \leqq\|\omega\|
$$

Also $\hat{n}$ and $\hat{r}_{B}$ are unit vectors,

Thus

$$
\left|\hat{n} \cdot \hat{r}_{B}\right| \leqq 1
$$

$$
\left|O_{1} \circ \omega\right| \leqq\|\omega\| \int_{B}\left|G_{0}\left(p, p_{B}\right)\right| d \sigma_{B}
$$

The inequality is strengthened if we insert the factor $\delta^{2} / r_{B}^{2}$ in the integrand whereupon we use (4.26) to write

$$
\left|O_{1} \circ \omega\right| \leqq \delta^{2} N\|\omega\|, \quad p \in \bar{V} .
$$

Again, this is true for all values and hence also for the maximum value of $\left|O_{1} \circ \omega\right|$. Thus, letting $M_{2}=\delta^{2} N$, we have

proving the lemma.

$$
\left|O_{1} \circ \omega\right| \leqq M_{2}\|\omega\|
$$


Lemma 4.5. $\|L\|<1$ for sufficiently small $|k|$.

Proof. Since $L$ and $L_{1}$ are linear,

$$
L=k L_{1} \text { implies }\|L\|=|k|\left\|L_{1}\right\| .
$$

Lemma 4.4 guarantees the existence of $M$ such that

$$
\left\|L_{1}\right\| \leqq M .
$$

Hence $\|L\| \leqq|k| \cdot M$. Choosing $|k|<1 / M$, we have proven the lemma.

Lemma 4.6. $\tilde{u}(p) \in W$.

Proof. $u$ is a scalar wave function, therefore $u \in C^{2}(\bar{V})$. Since $e^{-i k r}$ is analytic in $r$ and $r$ is continuously differentiable on $B, \tilde{u}=e^{-i k r} u$ satisfies the requirements that $\tilde{u} \in C^{2}(V)$ and $\tilde{u} \in C^{1}(\bar{V})$, hence $(4.4 \mathrm{a})$ is satisfied. Furthermore, scalar wave functions may be expanded in spherical harmonics, i.e.,

$$
u(p)=\sum_{n=0}^{\infty} h_{n}(k r) Y_{n}(\vartheta, \varphi), \quad r \geqq \delta
$$

where $h_{n}(k r)$ are spherical Hankel functions of the first kind. The fact that (4.4b) and (4.4c) are satisfied follows from this expansion and the definition of the Hankel functions. Details are found in [6].

We now are in a position to prove the main result.

Theorem 4.1. Let $B$ be a smooth, closed, finite surface in $E^{3}$ described by the equation $r_{B}=g(\vartheta, \varphi)$ such that $g$ is continuously differentiable for $0 \leqq \vartheta \leqq \pi$, $0 \leqq \varphi \leqq 2 \pi$, and $\hat{n} \cdot \hat{r}_{B}$ is uniformly Hölder continuous (equivalently, $\left[1+(\bar{\nabla} g)^{2}\right]^{-\frac{1}{2}}$ is uniformly Hölder continuous). If $u(p)$ is a scalar wave function for $V$, the exterior of $B$, then for $|k|$ sufficiently small $u(p)$ is given explicitly by the convergent expansion

$$
u(p)=e^{i k r} \sum_{n=0}^{\infty} L^{n} \circ u^{(0)}
$$

where

$$
\begin{gathered}
L \circ u^{(0)}=-2 i k \int_{V} \frac{G_{0}\left(p, p_{1}\right)}{r_{1}} \frac{\partial}{\partial r_{1}}\left[r_{1} u^{(0)}\left(p_{1}\right)\right] d v_{1}+i k \int_{B} G_{0}\left(p, p_{B}\right) \hat{n} \cdot \hat{r}_{B} u^{(0)}\left(p_{B}\right) d \sigma_{B}, \\
u^{(0)}(p)=-\int_{B} G_{0}\left(p, p_{B}\right) e^{-i k r_{B}} \frac{\partial}{\partial n} u\left(p_{B}\right) d \sigma_{B},
\end{gathered}
$$

$G_{0}\left(p, p_{B}\right)$ is the static Green's function whose normal derivative vanishes on $B$, and the normal is taken out of $V$.

Proof. Multiplying both sides of (4.39) by $e^{-i k r}$, we obtain

$$
\tilde{u}=\sum_{n=0}^{\infty} L^{n} \circ u^{(0)}
$$

Observe that $\tilde{u}, u^{(0)}$, and $u^{(N)}$ (the partial sums, 4.2) are in $W$ (Lemmas 4.1, 4.2 and 4.6). Thus $\left\|\tilde{u}-u^{(N)}\right\|$ is meaningful for any $N \geqq 0$. We shall prove the theorem 
by showing that for any $\varepsilon>0, \exists N_{0}(\varepsilon) \ni$

$$
\left\|\tilde{u}-u^{(N)}\right\|<\varepsilon \quad \text { provided } N>N_{0} .
$$

Theorem 3.2 shows that

$$
\tilde{u}=L \circ \tilde{u}+u^{(0)} \text {. }
$$

In addition, from the definition of $u^{(N)}$, we find

$$
u^{(N)}=\sum_{n=0}^{N} L^{n} \circ u^{(0)}, \text { which implies } u^{(N)}=L \circ u^{(N-1)}+u^{(0)} .
$$

With (4.41) and (4.42) it follows that

hence

$$
\tilde{u}-u^{(N)}=L^{N+1} \circ \tilde{u}
$$

$$
\left\|\tilde{u}-u^{(N)}\right\| \leqq\|L\|^{N+1}\|\tilde{u}\| .
$$

But Lemma 4.5 states that $\|L\|<1$ for $|k|$ sufficiently small and $\|\tilde{u}\|$ is bounded since $\tilde{u} \in W$ (Lemma 4.6). Therefore we may always make $\|L\|^{N+1}\|\tilde{u}\|$ as small as we wish by choosing $N$ large enough. Specifically for any $\varepsilon>0$

provided

$$
\|L\|^{N+1}\|\tilde{u}\|<\varepsilon
$$

This proves the theorem.

$$
N+1>\frac{\log \frac{\varepsilon}{\|\tilde{u}\|}}{\log \|L\|} .
$$

Representations of wave functions in two important special cases follow immediately.

Corollary 1. The Green's function of the second kind for the Helmholtz equation and the surface $B\left(\partial G_{k} / \partial n=0\right.$ on $\left.B\right)$ is

$$
G_{k}\left(p, p_{0}\right)=-\frac{e^{i k R\left(p, p_{0}\right)}}{4 \pi R\left(p, p_{0}\right)}+u\left(p, p_{0}\right)
$$

where $u\left(p, p_{0}\right)$ is defined explicitly in (4.39) with

$$
u^{(0)}\left(p, p_{0}\right)=-\frac{1}{4 \pi} \int_{B} G_{0}\left(p, p_{B}\right) e^{-i k r_{B}} \frac{\partial}{\partial n}\left[\frac{e^{i k R\left(p_{B}, p_{0}\right)}}{R\left(p_{B}, p_{0}\right)}\right] d \sigma_{B} .
$$

Corollary 2. The velocity potential $u^{t}$ when a plane acoustic wave is incident in a direction $\hat{\alpha}$ on a rigid surface $B\left(\partial u^{t} / \partial n=0\right.$ on $\left.B\right)$ is

$$
u^{t}(p)=e^{i k \vec{r} \cdot \hat{\alpha}}+u(p)
$$

where $u(p)$ is defined explicitly in (4.39) with

$$
u^{(0)}(p)=\int_{B} G_{0}\left(p, p_{B}\right) e^{-i k r_{B}} \frac{\partial}{\partial n}\left(e^{i k \overrightarrow{r_{B}} \cdot \hat{\alpha}}\right) d \sigma_{B} .
$$


We may also easily prove that a scalar wave function is uniquely determined by the values of its normal derivative on a boundary.

Theorem 4.2. If $u_{1}$ and $u_{2}$ are scalar wave functions for $V$ and

$$
\frac{\partial u_{1}}{\partial n}=\frac{\partial u_{2}}{\partial n} \quad \text { on } B
$$

then $u_{1}=u_{2}$ everywhere, $p \in \bar{V}$.

Proof. Assume $u_{1} \neq u_{2}$, and let $\tilde{u}_{1}=e^{-i k r} u_{1}$. With Theorem 3.2 we have

$$
\tilde{u}_{1}=L \circ \tilde{u}_{1}+u^{(0)}, \quad \tilde{u}_{2}=L \circ \tilde{u}_{2}+u^{(0)}
$$

where $u^{(0)}$ is the same in both equations since the normal derivatives are equal. Therefore

and

$$
\tilde{u}_{1}-\tilde{u}_{2}=L \circ\left(\tilde{u}_{1}-\tilde{u}_{2}\right) \text {, }
$$

$$
\left\|\tilde{u}_{1}-\tilde{u}_{2}\right\| \leqq\|L\|\left\|\tilde{u}_{1}-\tilde{u}_{2}\right\|
$$

Since $\left\|\tilde{u}_{1}-\tilde{u}_{2}\right\| \neq 0$, by assumption, we may divide, obtaining

which violates Lemma 4.5.

$$
1 \leqq\|L\|
$$

We conclude this section by pointing out the relation between the series representation of wave functions expressed in Theorem 4.1 and the usual low frequency expansion. If the boundary data is analytic in $k$, as is the case in Corollaries 1 and 2, then the low frequency expansion expresses the wave function as a power series in $k$, and the coefficients in such an expansion may be given in terms of the operators defined previously. Thus if

then we may write

$$
u^{(0)}(p)=\sum_{n=0}^{\infty} a_{n}(p) k^{n}
$$

$$
u(p)=\sum_{n=0}^{\infty} b_{n}(p) k^{n}
$$

where the $b_{n}$ 's are defined in terms of the $a_{n}$ 's as

$$
b_{n}=\sum_{m=0}^{n} \frac{(i r)^{n-m}}{(n-m) !} \sum_{l=0}^{m} L_{1}^{l} \circ a_{m-l} \text {. }
$$

This result is exactly the same as in the Dirichlet problem (with the definition of the operator changed), and details are given in [6].

\section{An Example - Scattering of a Plane Wave by a Sphere}

Both as a check and an illustration we apply the techniques described in the previous sections to a specific problem, scattering of a plane wave of sound by 
a rigid sphere. In this zase the exact result is known, and we are able to show not only that the iteration produces the correct result but how the $N^{\text {th }}$ iterate approximates the exact result.

The surface $B$ is now a sphere of radius $a$ whose center is taken as the origin of the coordinate system. The static Green's function of the second kind for the sphere is

where

$$
G_{0}\left(p, p_{1}\right)=-\frac{1}{4 \pi} \sum_{n=0}^{\infty}\left\{\frac{r_{<}^{n}}{r_{>}^{n+1}}+\frac{n a^{2 n+1}}{(n+1)\left(r r_{1}\right)^{n+1}}\right\} P_{n}(\cos \gamma)
$$

and

$$
\begin{aligned}
& r_{<}=\min \left(r, r_{1}\right), \\
& r_{>}=\max \left(r, r_{1}\right)
\end{aligned}
$$

$$
\cos \gamma=\hat{r} \cdot \hat{r}_{1}=\cos \vartheta \cos \vartheta_{1}+\sin \vartheta \sin \vartheta_{1} \cos \left(\varphi-\varphi_{1}\right)
$$

The incident field is a plane wave which, without loss of generality, is chosen as propagating down the $z$-axis, i.e.,

$$
u^{i}=e^{-i k \vec{r} \cdot \hat{i}_{z}}=e^{-i k z}=e^{-i k r \cos \vartheta} .
$$

The boundary values of interest are

$$
\frac{\partial u^{i}}{\partial n}=-\left.\frac{\partial}{\partial r} e^{-i k r \cos \vartheta}\right|_{r=a}=-k \sum_{n=0}^{\infty}(-i)^{n}(2 n+1) j_{n}^{\prime}(k a) P_{n}(\cos \vartheta)
$$

where $j_{n}$ is the spherical Bessel function and the prime denotes differentiation with respect to $k a$, i.e.,

$$
j_{n}^{\prime}(k a)=\frac{d}{d(k a)}\left[\left(\frac{\pi}{2 k a}\right)^{\frac{1}{2}} J_{n+\frac{1}{2}}(k a)\right] .
$$

The scattered field, $u$ (where $\partial u / \partial n=-\partial u^{i} / \partial n$ on $B$ ), is given by the methods described previously as

$$
u=\lim _{N \rightarrow \infty} e^{i k r} u^{(N)}
$$

where

$$
\begin{aligned}
u^{(N)}= & \sum_{n=0}^{N} L^{n} \circ u^{(0)} \\
L \circ u^{(0)}(p)= & \frac{i k}{2 \pi} \int_{V} \sum_{n=0}^{\infty}\left\{\frac{r_{<}^{n}}{r_{>}^{n+1}}+\frac{n}{n+1} \frac{a^{2 n+1}}{\left(r r_{1}\right)^{n+1}}\right\} \times \\
& \times P_{n}(\cos \gamma) \frac{1}{r_{1}} \frac{\partial}{\partial r_{1}}\left[r_{1} u^{(0)}\left(p_{1}\right)\right] d v_{1}+ \\
& +\frac{i k}{4 \pi} \int_{B} \sum_{n=0}^{\infty} \frac{2 n+1}{n+1} \frac{a^{n}}{r^{n+1}} P_{n}(\cos \gamma) u^{(0)}\left(p_{B}\right) d \sigma_{B}
\end{aligned}
$$


( $\cos \gamma$ always involves coordinates of $p$ and the integration variables) and

$$
\begin{aligned}
u^{(0)}(p)= & \frac{k e^{-i k a}}{4 \pi} \int_{B} \sum_{n=0}^{\infty} \frac{2 n+1}{n+1} \frac{a^{n}}{r^{n+1}} \times \\
& \times P_{n}(\cos \gamma) \sum_{m=0}^{\infty}(-i)^{m}(2 m+1) j_{m}^{\prime}(k a) P_{m}\left(\cos \vartheta_{B}\right) d \sigma_{B} .
\end{aligned}
$$

The orthogonality of the Legendre functions enables us to evaluate $u^{(0)}$ and the first few iterates. Omitting the details, we find these to be

$$
\begin{aligned}
u^{(0)}(p)= & k a e^{-i k a} \sum_{n=0}^{\infty} \frac{(2 n+1)}{(n+1)}(-i)^{n} \frac{a^{n+1}}{r^{n+1}} j_{n}^{\prime}(k a) P_{n}(\cos \vartheta) \\
u^{(1)}(p)= & k a e^{-i k a} \frac{a}{r} j_{0}^{\prime}(k a)(1+i k a)+ \\
& +k a e^{-i k a} \sum_{n=1}^{\infty} \frac{2 n+1}{n+1}(-i)^{n}(a / r)^{n+1} j_{n}^{\prime}(k a) P_{n}(\cos \vartheta)[1+i k a-i k r] \\
u_{u_{2}^{(2)}(p)=} & k a e^{-i k a} \frac{a}{r} j_{0}^{\prime}(k a)\left[1+i k a-(k a)^{2}\right]+ \\
& +k a e^{-i k a} \sum_{n=1}^{\infty} \frac{2 n+1}{n+1}(-i)^{n}(a / r)^{n+1} j_{n}^{\prime}(k a) \times \\
& \times P_{n}(\cos \vartheta)\left[1+i k(a-r)+k^{2} r a-\frac{(k r)^{2}(n-1)}{2 n-1}-\frac{(k a)^{2}\left(n^{2}+n-1\right)}{(n+1)(2 n-1)}\right] .
\end{aligned}
$$

The exact expression of $u$ is

$$
u(p)=-\sum_{n=0}^{\infty}(-i)^{n}(2 n+1) j_{n}^{\prime}(k a) P_{n}(\cos \vartheta) \frac{h_{n}(k r)}{h_{n}^{\prime}(k a)}
$$

where the prime again denotes differentiation with respect to $k a$ (see 5.4) and $h_{n}$ is a spherical Hankel function of the first kind. Explicitly

$$
h_{n}(z)=\frac{e^{i z}}{z} i^{-n-1} \sum_{m=0}^{n} \frac{(n+m) !}{(n-m) ! m !} \frac{1}{(-2 i z)^{m}} .
$$

With this definition we find that

$$
\begin{aligned}
\frac{h_{n}(k r)}{h_{n}^{\prime}(k a)}= & -k a e^{i k(r-a)}(a / r)^{n+1} \times \\
& \times \frac{\sum_{m=0}^{n} \frac{(2 n-m) !}{(n-m) ! m !}(-2 i k r)^{m}}{\sum_{m=0}^{n} \frac{(2 n-m) !}{(n-m) ! m !}(-2 i k a)^{m}(n+1-m-i k a)} .
\end{aligned}
$$

The ratio of the two polynomials, of degree $n$ in $k r$ in the numerator and $n+1$ in $k a$ in the denominator, may be re-expanded, for $k a$ sufficiently small, in 
ascending powers of $k$. Thus

$$
\frac{h_{n}(k r)}{h_{n}^{\prime}(k a)}=-k a \frac{e^{i k(r-a)}}{n+1}(a / r)^{n+1} \sum_{l=0}^{\infty} \alpha_{l} k^{l}, \quad n \geqq 0
$$

where the coefficients $\alpha_{l}$ are functions of $r, a$, and $n$. The first few have been found to be

$$
\begin{aligned}
& \alpha_{0}=1, \\
& \begin{aligned}
\alpha_{1} & =i a, \quad n=0, \\
& =i(a-r), \quad n>0, \\
\alpha_{2} & =-a^{2}, \quad n=0, \\
& =a r-\frac{n-1}{2 n-1} r^{2}-\frac{n^{2}+n-1}{(n+1)(2 n-1)} a^{2}, \quad n>0 .
\end{aligned}
\end{aligned}
$$

In terms of these expansions the exact result for $u$, equation (5.12), may be rewritten as

$$
u(p)=k a e^{i k(r-a)} \sum_{n=0}^{\infty}(-i)^{n} \frac{2 n+1}{n+1}(a / r)^{n+1} j_{n}^{\prime}(k a) P_{n}(\cos \vartheta) \sum_{l=0}^{\infty} \alpha_{l} k^{l} .
$$

If we denote by $u_{N}(p)$ the expression resulting from taking only the first $N$ terms in the expansions in $k$ in (5.17), that is,

$$
u_{N}=k a e^{i k(r-a)} \sum_{n=0}^{\infty}(-i)^{n} \frac{2 n+1}{n+1}(a / r)^{n+1} j_{n}^{\prime}(k a) P_{n}(\cos \vartheta) \sum_{l=0}^{N} \alpha_{l} k^{l},
$$

then we see that, for the values of $N$ computed,

$$
u_{N}=e^{i k r} u^{(N)}
$$

where the first few iterates, $u^{(N)}$, are given in (5.9) $-(5.11)$.

This could be pursued for more values of $N$ but there is little reason to do so. The terms explicitly evaluated not only serve as a check on the method but are sufficient to show how the iterates approximate the exact solution. Just as in the Dirichlet case, the $N^{\text {th }}$ iterate approximates the exact result just as $N$ terms of a power series expansion of the quotient of two polynomials approximates the quotient.

\section{Concluding Remarks}

The main result of this work has been the derivation of an explicit solution of the exterior Neumann problem in terms of given boundary data and the Green's function for Laplace's equation whose normal derivative vanishes on the surface. It should be noted that this work does not, as originally thought, constitute a proof of the existence of a wave function for arbitrary (differentiable) boundary data. The series of iterates in terms of which the solution is obtained (4.2) do form a convergent Cauchy sequence. However, in order to show that the limit is in the space which we have defined (Lemma 4.6) it is necessary to use 
the fact that this limit, in fact, exists. If the space were known to be complete, then this wouldn't be needed. That an existence proof can be constructed along these lines seems clear although some modification of the function space will be necessary. This work is now in progress.

Another point concerns the smoothness requirements of the boundary. The validity of the explicit representation of the solution of the Neumann problem has been proven only for smooth bodies. However, preliminary calculations for the circular disk support the hypothesis that the representation remains valid for bodies with edges. To prove this, however, will require a different definition of the norm. The pointwise convergence proven in Theorem 4.1 using the sup norm requires the wave functions to be twice differentiable on $B$. But it is known (e.g. BouWKamp [3], MeIXNer [8]) that wave functions associated with bodies with edges have singular derivatives. Thus, convergence of the iterates in these cases will have to be established in some other norm, presumably some modified form of $L^{2}(V)$. These remarks are equally applicable to the Dirichlet problem.

Finally, some comment is called for concerning the values of $k$ for which the series of iterates converges. While we have proven that the series converges for $|k|$ sufficiently small, that is, there exists some number $\alpha>0$ such that the series converges for $|k|<\alpha$, no indication was given as to how large $\alpha$ hence $|k|$, may be. If the boundary data is analytic in $k$, this problem of estimation is equivalent to finding the radius of convergence of the low frequency expansion (4.54). Such estimates are available only for special surfaces (e.g. DARLING \& SENIOR [4]) and the general problem remains unresolved.

Acknowledgment. The work reported in this paper was supported by the Air Force Cambridge Research Laboratories under Contract AF 19(628)-4328, the National Science Foundation under Grant GP-4581 and the Technical University of Denmark.

\section{References}

[1] Atkinson, F.V., On Sommerfeld's radiation condition. Philos. Mag. 40, Series 7, No. 305. $645-651$ (1949).

[2] BARrar, R.B., \& A.F. KAY, A Series Development of the Wave Equation in Powers of $1 / r$, Internal Memorandum Tech. Res. Group, Inc.

[3] Bouwkamp, C. J., Diffraction theory. Rep. Progr. Phys. 17, 35-100 (1954).

[4] Darling, D.A., \& T.B.A. Senior, Low frequency expansions for scattering by separable and nonseparable bodies. J. Acoust. Soc. Amer. 37, No. 2, 228-234 (1965).

[5] Kellogg, O.D. Foundations of Potential Theory. Berlin: Springer 1929.

[6] Kleinman, R. E., The Dirichlet problem for the Helmholtz equation. Arch. Rational Mech. Anal. 18, 205-229 (1965).

[7] LeIs, R., Ưber das Neumannsche Randwertproblem für die Helmholtzsche Schwingungsgleichung. Arch. Rational Mech. Anal. 2, 101-113 (1958).

[8] MexNer, J., Die Kantenbedingung in der Theorie der Beugung elektromagnetischer Wellen an vollkommen leitenden ebenen Schirmen. Ann. Phys. 6, 2-9 (1949).

[9] MüLLER, C., Zur Methode der Strahlungskapazität von H. Weyl. Math. Z. 56, 80-83 (1952).

[10] Noble, B., Integral Equation Perturbation Methods in Low Frequency Diffraction. From: Electromagnetic Waves (R. LANGER, ed.). Madison: The University of Wisconsin Press 1962.

[11] Sommerfeld, A., Die Greensche Funktion der Schwingungsgleichung. Jahresber. Math.Verein, 21, 309-353 (1912).

[12] Sommerfeld, A., Partial Differential Equations in Physics. New York: Academic Press 1949. 
[13] WeRnER, P., Randwertprobleme der mathematischen Akustik. Arch. Rational Mech. Anal. 10, 29-66 (1962).

[14] WeYL, H., Kapazität von Strahlungsfeldern. Math. Z. 55, 187-198 (1952).

[15] WIncox, C.H., A generalization of theorems of Rellich and Atkinson. Proc. Amer. Math. Soc. 7, 271-276 (1956).

[16] Wilcox, C.H., Spherical means and radiation conditions. Arch. Rational Mech. Anal. 3, 133-148 (1959).

Radiation Laboratory

University of Michigan

Ann Arbor

and

Laboratory of Electromagnetic Theory

Technical University of Denmark Lyngby

(Received May 16, 1966) 